\title{
PERIODIC 2-GRAPHS ARISING FROM SUBSHIFTS
}

\author{
DAVID PASK, IAIN RAEBURN ${ }^{\bowtie}$ and NATASHA A. WEAVER
}

(Received 28 October 2009)

\begin{abstract}
Higher-rank graphs were introduced by Kumjian and Pask to provide models for higher-rank CuntzKrieger algebras. In a previous paper, we constructed 2-graphs whose path spaces are ranktwo subshifts of finite type, and showed that this construction yields aperiodic 2-graphs whose $C^{*}$-algebras are simple and are not ordinary graph algebras. Here we show that the construction also gives a family of periodic 2-graphs which we call domino graphs. We investigate the combinatorial structure of domino graphs, finding interesting points of contact with the existing combinatorial literature, and prove a structure theorem for the $C^{*}$-algebras of domino graphs.
\end{abstract}

2000 Mathematics subject classification: primary 46L55; secondary 05C20, 22D25.

Keywords and phrases: 2-graph, subshift, graph algebra, crossed product, induced $C^{*}$-algebra.

\section{Introduction}

Higher-rank graphs (or $k$-graphs) are combinatorial objects which are higherdimensional analogues of directed graphs. They were invented by Kumjian and Pask [13] to provide combinatorial models for a family of higher-rank Cuntz-Krieger algebras studied by Robertson and Steger [26]. We now know that many important $C^{*}$-algebras can be realized as the $C^{*}$-algebras of higher-rank graphs, and there is a good deal of interest in understanding different classes of higher-rank graphs (see, for example, [3, 17, 31]).

Here we are interested primarily in 2-graphs. Intuitively, a 2-graph is a directed graph $\Lambda:=\left(\Lambda^{0}, \Lambda^{1}, r, s\right)$ in which the set $\Lambda^{1}$ is partitioned into a set $\Lambda^{e_{1}}$ of blue edges and set $\Lambda^{e_{2}}$ of red edges, together with a set $C$ of commuting squares

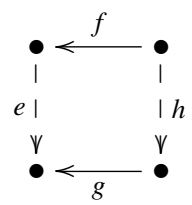

This research was supported by the Australian Research Council, and Natasha Weaver was supported by an Australian Postgraduate Award.

(C) 2010 Australian Mathematical Publishing Association Inc. 0004-9727/2010 \$16.00 
in which every blue-red path $g h$ and every red-blue path ef occur exactly once. We view $C$ as defining an equivalence relation on the path space $\Lambda^{*}$ which allows each path with $i$ blue edges and $j$ red edges to be rewritten in any chosen order of blue and red edges. If the square (1.1) belongs to $C$, for example, then $e f=g h$ as paths in $\Lambda$.

In [18], we constructed a family of 2-graphs whose infinite path spaces are ranktwo subshifts of finite type, as studied by Schmidt [30]. We found conditions which ensure that the $C^{*}$-algebras of these 2 -graphs are simple and purely infinite, and used results of Evans [6] to compute their $K$-theory in a wide range of examples. Here we consider a family of 2-graphs which we call domino graphs. They are also built using the construction in [18], but their $C^{*}$-algebras are definitely not simple. We find interesting connections with known combinatorial objects, such as necklaces and Lyndon words, and we analyse the structure of their $C^{*}$-algebras.

Our analysis uses two main operator-algebraic tools. We realize domino graphs as crossed products of an ordinary directed graph (or more strictly speaking, of the 1-graph it defines) by an action of $\mathbb{Z}$, as studied in recent work of Farthing et al. [7]; the results in [7] then imply that the $C^{*}$-algebra of a domino graph is the crossed product of an ordinary graph algebra by an action of $\mathbb{Z}$. Next, we observe that this action of $\mathbb{Z}$ on the graph algebra has large isotropy, and use a theorem of Olesen and Pedersen [16] to realize the crossed product as an induced $C^{*}$-algebra associated with a simple crossed product. This part of the analysis may be of some independent interest: we provide a new version of the Olesen-Pedersen theorem which gives a very specific isomorphism and which is easier to apply.

We begin in Section 2 with a quick review of $k$-graphs, emphasizing the connection to the intuitive description of 2-graphs given above. Then in Section 3 we discuss the general facts about crossed-product $C^{*}$-algebras which we need, and describe how they apply to the crossed-product graphs of [7]. In Section 4 we review the construction of [18], as it applies to dominos, and then analyse the structure of the resulting domino graphs using ideas from combinatorics which we discuss in Appendix A. The main result, Proposition 4.6, says that most domino graphs are crossed product 2-graphs of the sort studied in [7]. In Section 5 we combine the results of the preceding two sections to prove our structure theorem for the $C^{*}$-algebras of domino graphs, Theorem 5.1. In the final section we compute the $K$-theory of domino-graph algebras.

\section{2. $k$-graphs and their $C^{*}$-algebras}

A $k$-graph is a pair $(\Lambda, d)$ consisting of a countable category $\Lambda$ and a functor $d: \Lambda \rightarrow \mathbb{N}^{k}$, called the degree map, satisfying the factorization property: for every $\lambda \in \Lambda$ and $m, n \in \mathbb{N}^{k}$ with $d(\lambda)=m+n$, there exist unique elements $\mu, v \in \Lambda$ such that $d(\mu)=m, d(\nu)=n$ and $\lambda$ is the composition $\mu \nu$. We write $\Lambda^{0}$ for the set of objects, $\Lambda$ for the set of morphisms, and $s, r: \Lambda \rightarrow \Lambda^{0}$ for the domain and codomain maps, so that $\lambda$ and $\mu$ are composable exactly when $s(\lambda)=r(\mu)$. The factorization property implies that for every $v \in \Lambda^{0}$, there is a unique morphism $\mu$ such that 
$s(\mu)=r(\mu)$ and $d(\mu)=0$, namely the identity morphism $\iota_{v}$ at $v$. We use $v \mapsto \iota_{v}$ to identify $\Lambda^{0}$ with a subset of $\Lambda$. For $n \in \mathbb{N}^{k}$, we define $\Lambda^{n}:=\{\lambda \in \Lambda: d(\lambda)=n\}$, and refer to an element $\lambda$ of $\Lambda^{n}$ as a path of degree $n$ from $s(\lambda)$ to $r(\lambda)$.

In this paper we are concerned primarily with 2-graphs, and not much will be missed by a reader who assumes $k=2$ throughout. We visualize a 2 -graph $\Lambda$ using its skeleton, which is the directed bicoloured graph with vertex set $\Lambda^{0}$ and edge set $\Lambda^{e_{1}} \cup \Lambda^{e_{2}}$, where the elements $\beta$ of $\Lambda^{e_{1}}$ are blue edges from $s(\beta) \in \Lambda^{0}$ to $r(\beta) \in \Lambda^{0}$, and the elements of $\Lambda^{e_{2}}$ are red edges. (In print, solid curves represent blue edges and dashed curves represent red edges.) The skeleton thus consists of two directed graphs $B \Lambda:=\left(\Lambda^{0}, \Lambda^{e_{1}}, r, s\right)$ and $R \Lambda:=\left(\Lambda^{0}, \Lambda^{e_{2}}, r, s\right)$ with the same vertex set and different coloured edges.

The factorization property in a 2-graph $\Lambda$ is completely determined by the factorizations of paths of degree $(1,1)$ : writing $(1,1)=e_{1}+e_{2}$ and $(1,1)=e_{2}+e_{1}$ gives a bijection between the blue-red paths of length 2 and the red-blue paths of length 2 . We visualize a path of degree $(1,1)$ as a commuting square

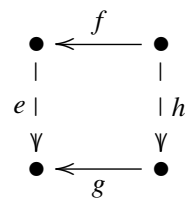

in which the bijection matches up the blue-red path $g h$ with the red-blue path $e f$, so that $g h=e f$ are the two factorizations of the path of degree $(1,1)$. Then the factorization property is determined by a family $C$ of commuting squares in which each red-blue and each blue-red path occurs exactly once (see [13, Section 6]). We then view paths of degree $(3,2)$ from $w$ to $v$, for example, as copies of the rectangle

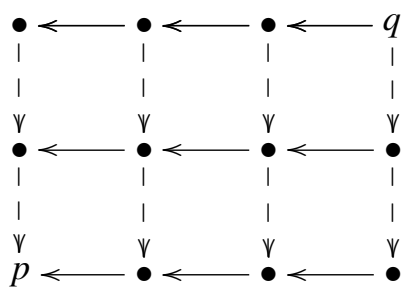

pasted round the blue-red graph, in such a way that $q$ lands on $w, p$ lands on $v$, and each constituent square belongs to $C$. Composing two paths involves adding squares from $C$ to fill out a larger rectangle, and [13, Section 6] says that there is only one way to do this.

Suppose that $\Lambda$ is a $k$-graph in which every vertex receives paths of all degrees. The $C^{*}$-algebra of $\Lambda$ is the universal $C^{*}$-algebra $C^{*}(\Lambda)$ generated by partial isometries $\left\{s_{\lambda}: \lambda \in \Lambda\right\}$ satisfying the following requirements:

(a) $\left\{s_{v}: v \in \Lambda^{0}\right\}$ are mutually orthogonal projections;

(b) $s_{\lambda} s_{\mu}=s_{\lambda \mu}$ whenever $s(\lambda)=r(\mu)$; 
(c) $s_{\lambda}^{*} s_{\lambda}=s_{s(\lambda)}$ for every $\lambda \in \Lambda$; and

(d) $s_{v}=\sum_{r(\lambda)=v, d(\lambda)=n} s_{\lambda} s_{\lambda}^{*}$ for every $v \in \Lambda^{0}$ and $n \in \mathbb{N}^{k}$.

For information about higher-rank graphs and their $C^{*}$-algebras, see [13] or [22].

\section{Crossed products of graph algebras}

In [7], Farthing et al. consider actions $\alpha: \mathbb{Z}^{l} \rightarrow$ Aut $\Lambda$ of $\mathbb{Z}^{l}$ on a $k$-graph $\Lambda$ and the induced actions $\tilde{\alpha}$ of $\mathbb{Z}^{l}$ on $C^{*}(\Lambda)$ [7, Proposition 3.1]. In this section, we establish some general properties of crossed products of the form $C^{*}(\Lambda) \times \tilde{\alpha} \mathbb{Z}^{l}$.

If $\alpha: G \rightarrow$ Aut $A$ is an action of a group on a $C^{*}$-algebra, then the crossed product $A \times_{\alpha} G$ is generated by a universal covariant representation $\left(i_{A}, i_{G}\right)$ in $M\left(A \times_{\alpha} G\right)$. We write $\pi \times U$ for the representation of $A \times{ }_{\alpha} G$ corresponding to a covariant representation $(\pi, U)$ of $(A, G, \alpha)$. Here we are only interested in actions of discrete groups, and then the map $i_{A}$ takes values in $A \times_{\alpha} G$, and the elements of the form $i_{A}(a) i_{G}(s)$ span a dense subspace of $A \times{ }_{\alpha} G$. When $G$ is abelian, the crossed product carries a canonical dual action $\hat{\alpha}$ of the dual group $\hat{G}$, which is characterized ${ }^{1}$ by $\hat{\alpha}_{\xi}\left(i_{A}(a) i_{G}(s)\right)=\xi(s) i_{A}(a) i_{G}(s)$.

To state our result about crossed products of the form $C^{*}(\Lambda) \times \tilde{\alpha} \mathbb{Z}^{l}$, we need one further concept. Suppose that $L$ is a closed subgroup of a compact group $K$, and $\alpha: L \rightarrow$ Aut $A$ is a continuous action. Then

$$
\operatorname{Ind}_{L}^{K}(A, \alpha)=\left\{f \in C(K, A): f(g h)=\alpha_{h}^{-1}(f(g)) \text { for } g \in K, h \in L\right\} .
$$

is a $C^{*}$-subalgebra of $C(K, A)$, called an induced $C^{*}$-algebra. (The extra assumption in [23, Section 6.3] that the function $s L \mapsto\|f(s)\|$ vanishes at $\infty$ on $K / L$ is automatic here because $K$ is compact.)

THEOREM 3.1. Suppose that $\alpha$ is an action of $\mathbb{Z}^{l}$ on a finite $k$-graph $\Lambda$. Then there are a cofinite subgroup $N$ of $\mathbb{Z}^{l}$ and an injective homomorphism $\beta: \mathbb{Z}^{l} / N \rightarrow$ Aut $\Lambda$ such that $\alpha_{m}=\beta_{m+N}$. Let $\Phi: C^{*}(\Lambda) \times_{\tilde{\alpha}} \mathbb{Z}^{l} \rightarrow C^{*}(\Lambda) \times_{\tilde{\beta}}\left(\mathbb{Z}^{l} / N\right)$ be the homomorphism such that $\Phi \circ i_{C^{*}(\Lambda)}=i_{C^{*}(\Lambda)}$ and $\Phi\left(i_{\mathbb{Z}^{l}}(m)\right)=i_{\mathbb{Z}^{l} / N}(m+N)$. Then there is an isomorphism $\Psi$ of $C^{*}(\Lambda) \times_{\tilde{\alpha}} \mathbb{Z}^{l}$ onto $\operatorname{Ind}_{N^{\perp}}^{\mathbb{T}^{l}}\left(C^{*}(\Lambda) \times_{\tilde{\beta}}\left(\mathbb{Z}^{l} / N\right), \widehat{\tilde{\beta}}\right)$ such that $\Psi(b)(z)=\Phi\left(\tilde{\alpha}_{z}^{-1}(b)\right)$.

Once we have shown the existence of $N$ and $\beta$, we will apply the following general result of Olesen and Pedersen [16].

THEOREM 3.2. Suppose that $H$ is a subgroup of a discrete abelian group $G$, and $\gamma$ is an action of $G / H$ on a $C^{*}$-algebra $A$. Let $q: G \rightarrow G / H$ be the quotient map, let $\beta:=\gamma \circ q$, and define $\Phi: A \times_{\beta} G \rightarrow A \times_{\gamma}(G / H)$ by $\Phi:=i_{A} \times\left(i_{G / H} \circ q\right)$. Then the formula $\Psi(b)(\xi)=\Phi\left(\hat{\beta}_{\xi}^{-1}(b)\right)$ defines an isomorphism $\Psi$ of $A \times \beta$ G onto Ind $_{H^{\perp}}^{\hat{G}}\left(A \times_{\gamma}(G / H), \hat{\gamma}\right)$.

\footnotetext{
${ }^{1}$ There is disagreement in the literature about the definition of the dual action: in [32], for example, $i_{G}(s)$ would be multiplied by $\overline{\xi(s)}=\xi\left(s^{-1}\right)$. It does not make a big difference, since $s \mapsto s^{-1}$ is an automorphism of the abelian group $G$, and induces an isomorphism of the two crossed products.
} 
With some effort, Theorem 3.2 can be deduced from [16, Corollary 2.5], which in turn is deduced from a chain of results involving both the 'restricted crossed products' of Dang Ngoc [2] and the 'twisted crossed products' of Green [9]. Since we are only interested in ordinary crossed products, we give a short direct proof, which is similar to that of [1, Theorem 2.1].

Proof. When we view $\xi \in H^{\perp}$ as a character of $G / H$, we have $\Phi \circ \hat{\beta}_{\xi}=\hat{\gamma}_{\xi} \circ \Phi$, and an easy calculation using this shows that $\Psi(b)$ belongs to the induced algebra.

Next we show that $\Psi$ is surjective. Since each generator $i_{A}(a) i_{G / H}(s+H)$ is just $\Phi\left(i_{A}(a) i_{G}(s)\right), \Phi$ is surjective. Thus, for each $\xi$, the elements $\Psi(b)(\xi)=$ $\Phi\left(\hat{\beta}_{\xi}^{-1}\left(\hat{\beta}_{\xi}(b)\right)\right)$ fill out $A \times_{\gamma}(G / H)$. Next, we observe that the integrated form $\left.i_{G}\right|_{H}: H \rightarrow U M\left(A \times{ }_{\gamma} G\right)$ maps $C^{*}(H)$ into the centre $Z\left(M\left(A \times{ }_{\gamma} G\right)\right)$, and hence by Fourier transformation gives a central action of $C(\hat{H})=C\left(\hat{G} / H^{\perp}\right)$ on $A \times{ }_{\gamma} G$. The algebra $C(\hat{H})=C\left(\hat{G} / H^{\perp}\right) \subset C(\hat{G})$ acts by pointwise multiplication on the induced algebra, and we claim that $\Psi$ is then $C(\hat{H})$-linear. To see this, consider a generator $\delta_{h}$ for $C^{*}(H)$ : since the Fourier transform of $\delta_{h}$ is the function $\epsilon_{h^{-1}}: \xi \mapsto$ $\overline{\xi(h)}$, we need to check that $\Psi\left(i_{G}(h) b\right)(\xi)=\overline{\xi(h)} \Psi(b)(\xi)$. For $b=i_{A}(a) i_{G}(s)$,

$$
\begin{aligned}
\Psi\left(i_{G}(h) b\right)(\xi) & =\Phi\left(\hat{\beta}_{\xi}^{-1}\left(i_{G}(h) i_{A}(a) i_{G}(s)\right)\right)=\Phi\left(\hat{\beta}_{\xi}^{-1}\left(i_{A}(a) i_{G}(h+s)\right)\right) \\
& =\Phi\left(\overline{\xi(h+s)} i_{A}(a) i_{G}(h+s)\right)=\overline{\xi(h) \xi(s)} i_{A}(a) i_{G / H}(s+H) \\
& =\overline{\xi(h)} \Phi\left(\hat{\beta}_{\xi}^{-1}(b)\right)=\overline{\xi(h)} \Psi(b)(\xi),
\end{aligned}
$$

as required. Thus $\Psi$ is $C(\hat{H})$-linear, as claimed. Now a partition-of-unity argument (as in [5, Lemma, p. 704], for example) shows that $\Psi$ is surjective.

To see that $\Psi$ is injective, it suffices to show that every irreducible representation $\pi \times U$ of $A \times \beta G$ factors through $\Psi$. Since $G$ is abelian, the operators $U_{h}$ for $h \in H$ commute with every $U_{s}$, and since $\beta_{h}$ is the identity on $A, U_{h}$ commutes with every $\pi(a)$ too. So $U$ maps $H$ into the commutant $(\pi \times U)(A \times G)^{\prime}$, which is $\mathbb{C} 1$ because $\pi \times U$ is irreducible, and there is a character $\theta$ of $H$ such that $U_{h}=\theta(h) 1$ for $h \in H$. By Pontryagin duality, there is a character $\chi \in \hat{G}$ such that $\left.\chi\right|_{H}=\theta$. Now $\chi^{-1} U$ factors through a unitary representation $V$ of $G / H$, and $(\pi, V)$ is covariant because $(\pi, U)$ is. Since $(\pi \times U)(b)=(\pi \times V)(\Psi(b)(\chi))$, the result follows.

Proof of Theorem 3.1. We begin by showing that Aut $\Lambda$ is finite. Aut $\Lambda$ consists of bijective functors $\alpha: \Lambda \rightarrow \Lambda$ which preserve the degree map: $d(\alpha(\lambda))=d(\lambda)$ for $\lambda \in \Lambda$. In particular, for each $n \in \mathbb{N}^{k}, \alpha$ is a bijection of $\Lambda^{n}$ onto $\Lambda^{n}$. Then define $\phi:$ Aut $\Lambda \rightarrow \bigoplus_{i=1}^{k}$ Perm $\Lambda^{e_{i}}$ by taking $\phi(\alpha)$ to be the tuple $\left(\left.\alpha\right|_{\Lambda^{e_{i}}}\right)_{i=1}^{k}$. Then we have

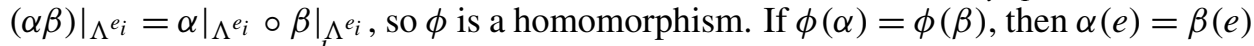
for all edges $e \in \bigcup_{i=1}^{k} \Lambda^{e_{i}}$, and it follows from the factorization property that $\alpha(\lambda)=\beta(\lambda)$ for all $\lambda$; thus $\phi$ is one-to-one. Since $\Lambda$ is a finite $k$-graph, $\bigoplus_{i=1}^{k}$ Perm $\Lambda^{e_{i}}$ is a finite group, and the injectivity of $\phi$ implies $\mid$ Aut $\Lambda \mid<\infty$. Let $N:=\operatorname{ker} \alpha$, and let $q: \mathbb{Z}^{l} \rightarrow \mathbb{Z}^{l} / N$ be the quotient map. Then there is a unique monomorphism $\beta: \mathbb{Z}^{l} / N \rightarrow$ Aut $\Lambda$ such that $\alpha=\beta \circ q$. Since Aut $\Lambda$ is finite, this 
implies in particular that $\mathbb{Z}^{l} / N$ is finite. The corresponding actions on $C^{*}(\Lambda)$ satisfy $\tilde{\alpha}=\tilde{\beta} \circ q$, so the result follows from Theorem 3.2.

COROLLARY 3.3. If $\alpha$ is an automorphism of a $k$-graph $\Lambda$ such that $\alpha^{n}$ is the identity, then $C^{*}(\Lambda) \rtimes_{\tilde{\alpha}} \mathbb{Z}$ is isomorphic to the mapping cylinder

$$
M(\gamma):=\left\{f:[0,1] \rightarrow C^{*}(\Lambda) \times_{\tilde{\alpha}}(\mathbb{Z} / n \mathbb{Z}): f \text { is continuous and } f(1)=\gamma(f(0)\}\right.
$$

associated with the generator $\gamma:=\hat{\tilde{\alpha}}_{e^{2 \pi i / n}}$ of the dual action.

Proof. The subgroup $N$ in Theorem 3.1 is $N=n \mathbb{Z}$, so $N^{\perp}$ is the group $C_{n}$ of $n$th roots of unity. But if $\theta: C_{n} \rightarrow$ Aut $B$ is an action on a $C^{*}$-algebra $B$, then the map $\phi: \operatorname{Ind}_{C_{n}}^{\mathbb{T}}(B, \theta) \rightarrow C([0,1], B)$ defined by $\phi(f)(t)=f\left(e^{-2 \pi i t / n}\right)$ is an isomorphism onto the mapping cylinder $M\left(\theta_{e^{2 \pi i / n}}\right)$.

The description of $C^{*}(\Lambda) \times \tilde{\alpha} \mathbb{Z}^{l}$ tells us that its primitive ideal space is the quotient of $\mathbb{T}^{l} \times \operatorname{Prim}\left(C^{*}(\Lambda) \times_{\tilde{\beta}}\left(Z^{l} / N\right)\right)$ by the diagonal action of $N^{\perp}$ (see [23, Proposition 6.16]). This is particularly interesting if $C^{*}(\Lambda)$ is simple, for then $\operatorname{Prim} C^{*}(\Lambda)=\{0\}$, and $\operatorname{Prim}\left(C^{*}(\Lambda) \times_{\tilde{\alpha}} \mathbb{Z}^{l}\right)$ is homeomorphic to $\mathbb{T}^{l} / N^{\perp}=\hat{N} \cong \mathbb{T}^{l}$ (because $N$, being a subgroup of a free abelian group, is itself free abelian). So we would like to know when $C^{*}(\Lambda) \times(\mathbb{Z} / n \mathbb{Z})$ is simple. For $k=1, \Lambda$ is the path space of a directed graph $E$ and $C^{*}(\Lambda)=C^{*}(E)$, and this question has an elegant answer.

We say that a directed graph $E$ is n-connected if for every pair of vertices $v, w \in E^{0}$, there is a path of length $n$ with source $v$ and range $w$. The following result is basically due to Katayama and Takehana [10].

PROPOSITION 3.4. Suppose that $E$ is a finite directed graph which is n-connected for some $n \geq 1$. If $\alpha$ is an automorphism of $E$ which is not the identity, then $\tilde{\alpha} \in C^{*}(E)$ is outer.

PROOF. We consider the Hilbert bimodule $X(E)$ over $c\left(E^{0}\right)$ constructed in [8] and [21, Section 8]: $X(E)$ is the completion of the bimodule $X_{0}=C\left(E^{1}\right)$ with actions and inner products

$$
(a \cdot f \cdot b)(e)=a(r(e)) f(e) b(s(e)) \quad \text { and } \quad\langle f, g\rangle_{A}(v)=\sum_{e \in E^{1}, s(e)=v} \overline{f(e)} g(e) .
$$

Indeed, since the graph can have no sources or sinks, the module $X_{0}$ will already be complete and is full. Then $\left\{k_{X(E)}\left(\delta_{e}\right), k_{A}\left(\delta_{v}\right)\right\}$ is a Cuntz-Krieger $E$ family in $\mathcal{O}(X(E))$, and the corresponding homomorphism of $C^{*}(E)$ into $\mathcal{O}(X(E))$ is an isomorphism (see [21, Example 8.13], for example). The elements $\left|s^{-1}(s(e))\right|^{-1 / 2} \delta_{e}$ form a Parseval frame for $X(E)$, so $X(E)$ is 'of finite type' as required in [10]. The $E^{0} \times E^{0}$ matrix $M$ constructed in [10, p. 497] is the vertex matrix of $E$, and the hypothesis of $n$-connectedness says precisely that $M^{n}(v, w)>0$ for every $v, w \in E^{0}$. So [10, Proposition 2.3] says that $C\left(E^{0}\right)$ is $X(E)$-aperiodic.

We define $\theta: C\left(E^{0}\right) \rightarrow C\left(E^{0}\right)$ by $\theta(f):=f \circ \alpha_{0}^{-1}$ and $U: X(E) \rightarrow X(E)$ by $U(x):=x \circ \alpha_{1}^{-1}$, and then $(\theta, U)$ satisfy the hypotheses (3.1) used in [10]. Since $\alpha$ 
is not the identity, the bijection $\alpha_{1}$ of $E^{1}$ is not the identity, and since automorphisms of $X(E)$ of the form $x \mapsto u x u^{*}$ are $C\left(E^{1}\right)$-linear, $U$ cannot have this form. So [10, Proposition 3.8] implies that $\alpha_{U}$ is outer. Since $\alpha_{U}\left(\delta_{e}\right)=\delta_{\alpha_{1}(e)}$ and $\theta\left(\delta_{v}\right)=\delta_{\alpha_{0}(v)}$, the isomorphism of $C^{*}(E)$ onto $\mathcal{O}(X(E))$ carries $\tilde{\alpha}$ into $\alpha_{U}$, and $\tilde{\alpha}$ is outer too.

REMARK 3.5. Since [10, Theorem 2.4] says that $X$-aperiodicity is equivalent to simplicity of the core $\mathcal{O}(X)^{\gamma}$, we should reconcile Proposition 3.4 with the results of Pask and Rho on simplicity of $C^{*}(E)^{\gamma}$ for finite $E$ [19]. (Examples in [19] show that the results do not extend to infinite $E$.) Pask and Rho define the period per $(v)$ of a vertex to be the greatest common divisor of $S(v):=\{|\mu|: s(\mu)=r(\mu)=v,|\mu|>1\}$; if $E$ is strongly connected, then all vertices have the same period, called the period of $E$. They prove in [19, Theorem 6.2] that $C^{*}(E)^{\gamma}$ is simple if and only if $E$ is strongly connected with period 1. This and Proposition 3.4 imply that a graph $E$ is strongly connected with period 1 if and only if $E$ is $n$-connected for some $n$. We should, of course, be able to see this directly.

If $E$ is $n$-connected, then we can get from a vertex $v$ to each of its neighbours by a path of length $n$, and hence back to $v$ by one of length $n+1$. Hence $v$ admits return paths of length $n+k$ for every $k \in \mathbb{N}$, and $\operatorname{per}(v)=1$. If $E$ is strongly connected with period 1 , then there exists $m$ such that the subsemigroup $S(v)$ of $\mathbb{N}$ contains $m+\mathbb{N}$. If $r$ is the diameter of $E$, then we can get from any vertex to any other by a path of length $m+2 r$ : choose a path through $v$ of length $s \leq 2 r$, and insert a return path at $v$ of length $m+(2 r-s)$.

COROLlary 3.6. Suppose that $\alpha$ is an action of $\mathbb{Z}^{l}$ on an $n$-connected directed graph $E$ which does not consist of a single loop, and $\beta$ is the injection $\beta: \mathbb{Z}^{l} / N \rightarrow$ Aut $E$ of a finite quotient of $\mathbb{Z}^{l}$ such that $\alpha=\beta \circ q$. Then $C^{*}(E) \times{ }_{\tilde{\beta}}\left(\mathbb{Z}^{l} / N\right)$ is simple, and the primitive ideal space of $C^{*}(E) \times \tilde{\alpha} \mathbb{Z}^{l}$ is homeomorphic to $\mathbb{T}^{l}$.

PROOF. Theorem 3.1 tells us that there is an action $\beta$ of a finite quotient $\mathbb{Z}^{l} / N$ such that $\beta_{m+N}$ is nontrivial whenever $m+N \neq N$. So Proposition 3.4 imples that $\tilde{\beta}_{m+N}$ is outer for every nonzero $m+N$. The $n$-connectedness hypothesis implies that $E$ cannot consist of single cycle of length greater than 1 , so the simplicity theorem for graph algebras (or the original theorem of Cuntz and Krieger) tells us that $C^{*}(E)$ is simple. Thus Kishimoto's [11] Theorem 3.1 implies that $C^{*}(E) \times{ }_{\tilde{\beta}}\left(\mathbb{Z}^{l} / N\right)$ is simple. Now the result follows from the isomorphism of our Theorem 3.1 and the description of the primitive ideal space of induced algebras in [23, Proposition 6.61].

Although they are not needed here, we ask whether there are similar criteria for the simplicity of $C^{*}(\Lambda) \times_{\tilde{\beta}}\left(\mathbb{Z}^{l} / N\right)$ when $\Lambda$ has rank greater than one. Robertson and Sims have given us good criteria for the simplicity of $C^{*}(\Lambda)$ [25], and of course Kishimoto's theorem is still valid, so what is missing is a version of the KatayamaTakehana theorem (that is, Proposition 3.4) for $k$-graphs. It is not obvious, though, that the methods used in [10] will carry over. 


\section{Domino graphs}

The 2-graphs in [18] are associated with basic data $(T, q, t, w)$ consisting of:

- a tile $T$, which is a hereditary $(j \in T$ and $0 \leq i \leq j$ imply $i \in T)$ subset of $\mathbb{N}^{2}$ with finite cardinality $|T|$;

- an alphabet $\{0,1, \ldots, q-1\}$, where $q \geq 2$ is an integer; we view the alphabet as a commutative ring by identifying it with $\mathbb{Z} / q \mathbb{Z}$ in the obvious way;

- an element $t$ of the alphabet, called the trace; and

- a function $w: T \rightarrow\{0,1, \ldots, q-1\}$, called the rule.

Provided certain values of $w$ are invertible elements of $\mathbb{Z} / q \mathbb{Z}$, Theorem 3.4 of [18] tells us how to construct a 2-graph $\Lambda=\Lambda(T, q, t, w)$ from this data.

A domino is a tile of the form $T=\left\{0, e_{1}, 2 e_{1}, \ldots,(n-1) e_{1}\right\}$, which is determined by $|T|:=n$. In this paper we write $(n, q, t)$ for the basic data consisting of the domino of cardinality $n$, alphabet $\mathbb{Z} / q \mathbb{Z}$ and trace $t$, and we always take the rule $w$ to be the constant function 1. Then every value of $w$ is invertible, and [18, Theorem 3.4] gives a 2-graph $\Lambda=\Lambda(n, q, t)$, which we call a domino graph.

Each 2-graph $\Lambda$ of [18] is finite, has no sources [18, Proposition 3.2], is strongly connected in the sense that $u \Lambda v$ is nonempty for all $u, v \in \Lambda^{0}$ [18, Proposition 5.3], and has at most one edge of each colour between any pair of vertices [18, Proposition 3.5(b)]. So domino graphs have all these properties.

We now briefly recall the construction of [18, Section 2] as it applies to domino graphs. We picture the domino $T$ with $|T|=n$ as a row of $n$ boxes; for example, we draw the domino with $n=6$ as , and we picture a vertex in $\Lambda$ as a copy of $T$ in which each box is filled with an element of the alphabet so that the sum of the entries is $t \bmod q$. Formally,

$$
\Lambda(n, q, t)^{0}=\left\{v: T \rightarrow \mathbb{Z} / q \mathbb{Z}: \sum_{i \in T} v(i)=t \bmod q\right\},
$$

and in $\Lambda(6,2,0)$, for example, the function $v: T \rightarrow \mathbb{Z} / 2 \mathbb{Z}$ with

$$
v(0)=v\left(3 e_{1}\right)=0, \quad v\left(e_{1}\right)=v\left(2 e_{1}\right)=v\left(4 e_{1}\right)=v\left(5 e_{1}\right)=1
$$

gives a vertex pictured as \begin{tabular}{|l|l|l|l|l|l|}
\hline 0 & 1 & 1 & 0 & 1 & 1 \\
\hline
\end{tabular}

To describe the paths we need some notation. For $S \subset \mathbb{Z}^{2}$ and $m \in \mathbb{Z}^{2}$, we define $S+m:=\{i+m: i \in S\}$ and $T(m):=\bigcup_{0 \leq l \leq m} T+l$. If $f: S \rightarrow \mathbb{Z} / q \mathbb{Z}$ is a function defined on a subset $S$ of $\mathbb{N}^{2}$ containing $T+l$, then we define $\left.f\right|_{T+l}: T \rightarrow \mathbb{Z} / q \mathbb{Z}$ by

$$
\left.f\right|_{T+l}(i)=f(i+l) \quad \text { for } i \in T \text {. }
$$

A path of degree $m$ is a function $\lambda: T(m) \rightarrow \mathbb{Z} / q \mathbb{Z}$ such that $\left.\lambda\right|_{T+l}$ is a vertex for every $0 \leq l \leq m$; then $\lambda$ has source $s(\lambda)=\left.\lambda\right|_{T+m}$ and range $r(\lambda)=\left.\lambda\right|_{T}$. Then $\Lambda^{m}$ denotes the set of paths of degree $m$ and $\Lambda^{*}=\bigcup_{m \geq 0} \Lambda^{m}$. In pictures, paths in $\Lambda^{*}$ 
are block diagrams covered by translates of $T$, filled in so that each translate is a valid vertex. For example, in $\Lambda(6,2,0)$, the diagram

$$
\mu=\begin{array}{|l|l|l|l|l|l|l|l|l|}
\hline 0 & 0 & 0 & 0 & 0 & 0 & 0 & 0 & 0 \\
\hline 0 & 0 & 0 & 1 & 1 & 0 & 0 & 0 & 0 \\
\hline 0 & 1 & 1 & 0 & 1 & 1 & 0 & 1 & 1 \\
\hline
\end{array}
$$

represents a path $\mu$ of degree $(3,2)$ from $s(\mu)=$\begin{tabular}{|l|l|l|l|l|l|}
\hline 0 & 0 & 0 & 0 & 0 & 0 \\
(the upper right-
\end{tabular} hand translate of $T$ ) to $r(\mu)=$\begin{tabular}{l|l|l|l|l|l|l}
0 & 1 & 1 & 0 & 1 & 1 \\
\hline
\end{tabular}

The paths $\Lambda^{*}$ form a category with the composition defined in [18, Proposition 3.2]: we say that $\mu \in \Lambda^{m}$ and $\nu \in \Lambda^{p}$ are composable if $s(\mu)=r(\nu)$ and then there exists a unique path $\lambda \in \Lambda^{m+p}$ satisfying $\left.\lambda\right|_{T(m)}=\mu$ and $\lambda \mid T(n)-m=v$. So if $\mu$ is the path in $(4.1)$ and $v$ is the path of degree $(2,1)$ with diagram

$v=$\begin{tabular}{|l|l|l|l|l|l|l|l|}
\hline 1 & 1 & 1 & 1 & 1 & 1 & 1 & 1 \\
\hline 0 & 0 & 0 & 0 & 0 & 0 & 0 & 0 \\
\hline
\end{tabular}

then $s(\mu)=r(\nu)$, and $\mu \nu$ is found by filling in the empty squares in the diagram

$$
\mu=\left(\begin{array}{|l|l|l|l|l|l|l|l|l|l|l|l|}
\hline \\
\hline 0 & 0 & 0 & 0 & 0 & 0 & 0 & 0 & 0 & 0 & 0 \\
\hline 0 & 0 & 0 & 1 & 1 & 0 & 0 & 0 & 0 & & \\
\hline 0 & 1 & 1 & 0 & 1 & 1 & 0 & 1 & 1 & \\
\hline
\end{array}\right.
$$

The proof of [18, Proposition 3.2] shows that we can do this in such a way that the entry for each empty box is completely determined by previous steps. With $d: \Lambda \rightarrow \mathbb{N}^{2}$ defined by $d(\lambda)=m$ for $\lambda \in \Lambda^{m}, \Lambda=\Lambda(n, q, t)$ is a 2-graph [18, Theorem 3.4].

REMARK 4.1. Theorem 4.1 of [18] says that the two-sided infinite path space $\Lambda(n, q, 0)^{\Delta}$ is conjugate to the two-dimensional shift of finite type with underlying space

$$
\Omega=\left\{f: \mathbb{Z}^{2} \rightarrow \mathbb{Z} / q \mathbb{Z}:\left.\sum_{i \in T} f\right|_{T+l}(i)=0 \bmod q \text { for all } l \in \mathbb{Z}^{2}\right\} .
$$

The shift $\Omega$ corresponds to the model $R_{2}^{q} /(g)$ of [12, Section 3] in which $g$ is the cyclotomic polynomial $g_{T}\left(u_{1}, u_{2}\right)=1+u_{1}+\cdots+u_{1}^{n-1}$. Theorem 6.5(2) of [30] implies that $\Omega$ is a nonmixing shift, whereas the shifts associated with the graphs in [18, Sections 5 and 6] are mixing.

We can explicitly describe the skeletons of domino graphs as follows.

Proposition 4.2. Suppose that $\Lambda=\Lambda(n, q, t)$ is a domino 2-graph. Then:

(1) the red graph $R \Lambda$ is the complete directed graph $K_{q^{n-1}}$, so that $\left|v \Lambda^{e_{2}} u\right|=1$ for all $u, v \in \Lambda^{0}$;

(2) $\quad \Lambda^{0}$ can be identified with the set $A_{t}^{n}$ of words of length $n$ with trace $t$ mod $q$ over the alphabet $A=\mathbb{Z} / q \mathbb{Z}$; 
(3) the blue graph $B \Lambda$ consists of disjoint cycles whose lengths divide $n$, and correspond to the necklaces of $A_{t}^{n}$ discussed in Appendix A. For each divisor $d$ of $n$, the number $h_{d}$ of blue cycles of length $d$ in $\Lambda$ is

$$
h_{d}=\sum_{\substack{s \in \mathbb{Z} / q \mathbb{Z}, d^{-1} s n \equiv t \bmod q}} L_{q}(d, s),
$$

where $L_{q}(d, s)$ is the number of Lyndon words of $A_{s}^{d}$ described in (A.2).

PROOF. Proposition 3.5 of [18] says that $\left|\Lambda^{0}\right|=q^{n-1}$ and $\left|v \Lambda^{e_{2}}\right|=\left|\Lambda^{e_{2}} v\right|=q^{n-1}$ for all $v \in \Lambda^{0}$. Since $\left|v \Lambda^{e_{2}} u\right| \leq 1$ for all $u, v \in \Lambda^{0}$, this forces $\left|v \Lambda^{e_{2}} u\right|=1$ for all $u, v \in \Lambda^{0}$. For (2), we identify each vertex $v \in \Lambda^{0}$ with its image written as the concatenation $v(0) v\left(e_{1}\right) v\left(2 e_{1}\right) \cdots v\left((n-1) e_{1}\right)$, which is an element of the set $A_{t}^{n}$. For (3), $B \Lambda$ must consist of disjoint cycles since $\left|v \Lambda^{e_{1}}\right|=\left|\Lambda^{e_{1}} v\right|=1$ for all $v \in \Lambda^{0}$ by [18, Proposition 3.5]. The equivalence classes of $A_{t}^{n}$ under the rotation $\rho$ defined in Appendix A are called necklaces. Under the identification $\Lambda^{0}=A_{t}^{n}$, the cycles in $B \Lambda$ correspond to the necklaces of $A_{t}^{n}$, and the length of each cycle equals the period of the corresponding necklace which must divide $n$. Suppose $[a]$ is a necklace with period $d$ and let $b$ be its Lyndon subword. Then since $t=\operatorname{trace}(a)=\operatorname{trace}\left(b^{n / d}\right)$, we have $n / d \times \operatorname{trace}(b)=t$. So the number of $d$-cycles in $B \Lambda$ equals the number of Lyndon words of length $d$ whose trace $s \in \mathbb{Z} / q \mathbb{Z}$ satisfies $d^{-1} s n \equiv t \bmod q$.

EXAmple 4.3. Suppose $\Lambda=\Lambda(6,2,0)$. The divisors of 6 are $d_{1}=1, d_{2}=2, d_{3}=3$, $d_{4}=6$. The constants of Proposition 4.2(3) are $h_{1}=2, h_{2}=0, h_{3}=2, h_{4}=4$, and so $B \Lambda$ consists of two 1-cycles, two 3-cycles and four 6-cycles. Identifying the vertex set of $\Lambda(6,2,0)$ with the set $A_{0}^{6}$ of words of length 6 over $A=\{0,1\}$ with trace $0 \bmod 2$, the cycles in $B \Lambda$ correspond to the necklaces of $A_{0}^{6}$ which are listed in the first column of Table A1 in Appendix A. For example, the 3-cycle in the diagram below corresponds to the necklace [011011] with period 3 of (A.1).

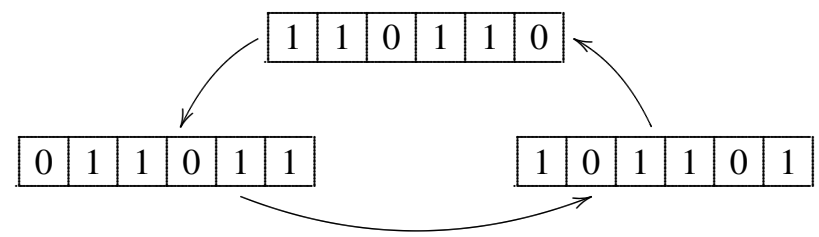

LEMma 4.4. Suppose that $\Lambda=\Lambda(n, q, t)$ is a domino 2-graph. Define $\sigma: \Lambda^{0} \rightarrow \Lambda^{0}$ by

$$
\sigma(v)=s(e) \quad \text { where } e \in v \Lambda^{e_{1}} .
$$

Then $\sigma$ is a bijection. Further, $\sigma$ is the identity if $n=1$ or $(n, q, t)=(2,2,0)$, and otherwise $\sigma$ has order $n$.

Proof. Under the identification $\Lambda^{0}=A_{t}^{n}$ of Proposition 4.2(2), $\sigma$ corresponds to $\rho$ defined in Appendix A. There is a unique blue edge entering and leaving each vertex by Proposition 4.2(3), and so $\sigma$ is a bijection. 
The order of a vertex $v \in \Lambda^{0}$ under $\sigma$ is the length of the cycle on which $v$ lies. The blue graph of $\Lambda(1, q, t)$ has only one vertex $t$ and one blue edge \begin{tabular}{|l|l}
$t$ & $t$
\end{tabular} , which is a

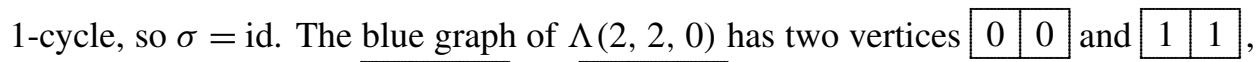

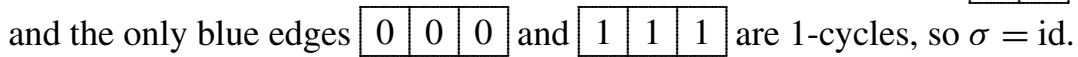

Otherwise, note that $\sigma$ has order $n$ if and only if $B \Lambda$ contains an $n$-cycle. If $t \neq 0$, then the vertex $v$ with $v(0)=t$ and $v\left(i e_{1}\right)=0$ for $1 \leq i \leq n-1$ lies on an $n$-cycle. If $t=0$, then the vertex $u$ with $u(0)=u\left(e_{1}\right)=1$, and $u\left(i e_{1}\right)=0$ for $2 \leq i \leq n-1$ lies on an $n$-cycle.

The permutation $\sigma$ satisfies $\sigma(r(e))=s(e)$, and hence moves against the direction of the edges. For example, in $\Lambda(6,2,0), \sigma\left(\begin{array}{|l|l|l|l|l|l}0 & 1 & 1 & 0 & 1 & 1 \\
\hline\end{array}\right)=$\begin{tabular}{|l|l|l|l|l|l|}
\hline 1 & 1 & 0 & 1 & 1 & 0 \\
\hline
\end{tabular}

Let $E$ and $F$ be 1-graphs. The product graph $(E \times F, d)$ is the 2-graph consisting of the product category $E \times F$ with the degree map $d\left(\lambda_{1}, \lambda_{2}\right)=\left(d\left(\lambda_{1}\right), d\left(\lambda_{2}\right)\right)$. The following lemma says that the domino graphs for which $\sigma$ is the identity are the only ones which are product graphs.

LEMMA 4.5. The domino graph $\Lambda(n, q, t)$ is a product graph if and only if $n=1$, in which case $\Lambda \cong K_{1} \times K_{1}$, or $(n, q, t)=(2,2,0)$, in which case $\Lambda \cong K_{1} \times K_{2}$.

PROOF. Suppose that $\Lambda$ is a product graph $E \times F$. Then

$$
\Lambda^{e_{2}}=(E \times F)^{e_{2}}=\left\{(v, f): v \in E^{0}, f \in F^{1}\right\}
$$

with $s(v, f)=(v, s(f))$ and $r(v, f)=(v, r(f))$. Since Proposition 4.2 implies that $R \Lambda$ is connected, we must have $\left|E^{0}\right|=1$. Then $\left|F^{0}\right|=q^{n-1}$ since

$$
\left|E^{0}\right| \times\left|F^{0}\right|=\left|E^{0} \times F^{0}\right|=\left|(E \times F)^{0}\right|=\left|\Lambda^{0}\right|=q^{n-1},
$$

and so $B \Lambda$ has $q^{n-1}$ copies of (the underlying directed graph of) $E$.

Recall from [18, Proposition 3.5(b)] that there is at most one edge of each colour between any pair of vertices in the skeleton of $\Lambda$. So since $E$ has only one vertex, there is either no edge or exactly one edge, which has to be a loop. If $E$ had no edge, then $B \Lambda$ would have no edges, so $E$ must be $E=K_{1}$. Since $B \Lambda$ has $q^{n-1}$ copies of $E=K_{1}$, there is a blue loop at each vertex. Thus $\sigma=\mathrm{id}$ and Lemma 4.4 implies that either $n=1$ or $(n, q, t)=(2,2,0)$. If $n=1$, then Proposition 4.2 implies that $h_{1}=1$ and $F=K_{1}$; if $(n, q, t)=(2,2,0)$, then $h_{1}=2$ and $F=K_{2}$.

Suppose that $\alpha$ is an action of $\mathbb{Z}^{l}$ on a $k$-graph $\Lambda$. Farthing et al. [7] constructed a crossed product $(k+l)$-graph $\Lambda \times_{\alpha} \mathbb{Z}^{l}$, whose underlying set is the cartesian product $\Lambda \times \mathbb{N}$, and which has degree map ${ }^{1}$ defined by $d(\lambda, m):=(m, d(\lambda))$, range and source maps defined by $r(\lambda, m):=(r(\lambda), 0)$ and $s(\lambda, m):=\left(\alpha^{-m}(s(\lambda)), 0\right)$, and composition defined by $(\mu, m)(\nu, n)=\left(\mu \alpha^{m}(v), m+n\right)$ when $s(\mu, m)=r(v, n)$.

${ }^{1}$ This is slightly different from the definition in [7], where the degree map is defined by $d(\lambda, m):=$ $(d(\lambda), m)$. The change has the effect of repainting the red edges blue and vice versa; we have made the change to ensure that the isomorphism of Proposition 4.6 matches red edges with red edges. 
One of the main theorems in [7] says that the graph algebra $C^{*}\left(\Lambda \times_{\alpha} \mathbb{Z}^{l}\right)$ is isomorphic (in a very concrete way) to the $C^{*}$-algebraic crossed product $C^{*}(\Lambda) \times \tilde{\alpha} \mathbb{Z}^{l}$ which we considered in Section 3 [7, Theorem 3.5]. In our application of these ideas, we go the other way: we recognize that our domino graphs are crossed products of the form $R \Lambda \times \mathbb{Z}$, and then we use properties of crossed product $C^{*}$-algebras to study the $C^{*}$-algebras of domino graphs. The action $\alpha$ is defined using the permutation $\sigma$ introduced in Lemma 4.4.

Proposition 4.6. Suppose that $\Lambda=\Lambda(n, q, t)$ is the domino graph associated with basic data $(n, q, t)$ with $n \geq 2$. For each $e \in \Lambda^{e_{2}}$, there is a unique edge $\sigma_{1}(e)$ in $\Lambda^{e_{2}}$ from $\sigma(s(e))$ to $\sigma(r(e))$, and then $\sigma_{1}: \Lambda^{e_{2}} \rightarrow \Lambda^{e_{2}}$ is a bijection. The pair $\left(\sigma, \sigma_{1}\right)$ is an automorphism of $R \Lambda$. Let $\alpha$ be the action of $\mathbb{Z}$ on $R \Lambda$ generated by $\left(\sigma^{-1}, \sigma_{1}^{-1}\right)$. Then $\Lambda$ is isomorphic to the crossed product $R \Lambda \times_{\alpha} \mathbb{Z}$.

PROOF. Since $R \Lambda$ is complete (by Proposition 4.2), there is exactly one red edge between every pair of vertices in $\Lambda^{0}$, so $\sigma_{1}$ is well defined. To see that $\sigma_{1}$ is surjective, let $f \in \Lambda^{e_{2}}$. Then there exist unique blue edges $f_{1}, f_{2}$ with $s\left(f_{1}\right)=r(f)$ and $s\left(f_{2}\right)=s(f)$; take $e$ to be the unique edge from $r\left(f_{2}\right)$ to $r\left(f_{1}\right)$, and then we have $\sigma(r(e))=s\left(f_{1}\right)=r(f), \sigma(s(e))=s\left(f_{2}\right)=s(f)$ and $\sigma_{1}(e)=f$. To see that $\sigma_{1}$ is injective, suppose that $\sigma_{1}(e)=\sigma_{1}(h)$. Then

$$
s(e)=\sigma^{-1}\left(s\left(\sigma_{1}(e)\right)\right)=\sigma^{-1}\left(s\left(\sigma_{1}(h)\right)\right)=s(h) \quad \text { and } \quad r(e)=r(h),
$$

and since there is exactly one red edge between two given vertices, we must have $e=h$. The pair $\left(\sigma, \sigma_{1}\right)$ is an automorphism of $R \Lambda$ since $\sigma$ and $\sigma_{1}$ are bijections and we have $s\left(\sigma_{1}(e)\right)=\sigma(s(e))$ and $r\left(\sigma_{1}(e)\right)=\sigma(r(e))$ by definition.

We build a coloured graph isomorphism $\phi$ from the skeleton of $\Lambda$ to the skeleton of $R \Lambda \times_{\alpha} \mathbb{Z}$ and prove that it preserves commuting squares. Then by [13, Section 6], $\phi$ extends uniquely to a 2-graph isomorphism, and the result follows.

We define

$$
\left\{\begin{array}{lll}
\phi_{0}: \Lambda^{0} \rightarrow\left(R \Lambda \times_{\alpha} \mathbb{Z}\right)^{0} & \text { by } \phi_{0}(v)=(v, 0) & \text { for } v \in \Lambda^{0} \\
\phi_{1}: \Lambda^{e_{1}} \rightarrow\left(R \Lambda \times_{\alpha} \mathbb{Z}\right)^{e_{1}} & \text { by } \phi_{1}(\beta)=(r(\beta), 1) & \text { for } \beta \in \Lambda^{e_{1}}, \\
\phi_{2}: \Lambda^{e_{2}} \rightarrow\left(R \Lambda \times_{\alpha} \mathbb{Z}\right)^{e_{2}} & \text { by } \phi_{2}(\rho)=(\rho, 0) & \text { for } \rho \in \Lambda^{e_{2}}
\end{array}\right.
$$

Then $\phi_{0}$ and $\phi_{2}$ are bijections because $\left(R \Lambda \times_{\alpha} \mathbb{Z}\right)^{0}=\Lambda^{0} \times\{0\}$ and $\left(R \Lambda \times_{\alpha} \mathbb{Z}\right)^{e_{2}}=$ $\Lambda^{e_{2}} \times\{0\}$. To see that $\phi_{1}$ is a bijection, note that

$$
\left(R \Lambda \times_{\alpha} \mathbb{Z}\right)^{e_{1}}=\left\{(r(\beta), 1): \beta \in \Lambda^{e_{1}}\right\}=\left\{(v, 1): v \in \Lambda^{0}\right\}
$$

since $\left|v \Lambda^{e_{1}}\right|=1$ for all $v \in \Lambda^{0}$, and let $\beta \in \Lambda^{e_{1}}$. Then $\phi_{1}(\beta)=(r(\beta), 1)$ is the unique edge with range $r(r(\beta), 1)=(r(\beta), 0)$ and source $s(r(\beta), 1)=\left(\alpha^{-1}(r(\beta)), 0\right)=$ $(s(\beta), 0)$. Hence $\phi_{1}$ is a bijection. So $\phi=\left(\phi_{0}, \phi_{1}, \phi_{2}\right)$ is an isomorphism and it remains to show that it preserves commuting squares.

Every commuting square $\lambda \in \Lambda^{(1,1)}$ is uniquely determined by the red edge $\lambda\left(0, e_{2}\right)=\left.\lambda\right|_{T\left(e_{2}\right)}$. To see this, let $\rho \in \Lambda^{e_{2}}$. There are unique blue edges $\beta_{1}$ and $\beta_{2}$ 
with $r\left(\beta_{1}\right)=r(\rho)$ and $s\left(\beta_{2}\right)=s(\rho)$, and we then have $s\left(\beta_{1}\right)=\alpha^{-1}\left(r\left(\beta_{1}\right)\right)$ and $s\left(\beta_{2}\right)=\alpha^{-1}\left(r\left(\beta_{2}\right)\right)$. There is a unique red edge from $s\left(\beta_{2}\right)$ to $s\left(\beta_{1}\right)$, and it must be $\alpha^{-1}(\rho)$ since

$$
r\left(\alpha^{-1}(\rho)\right)=\alpha^{-1}(r(\rho))=\alpha^{-1}\left(r\left(\beta_{1}\right)\right)=s\left(\beta_{1}\right)
$$

and

$$
s\left(\alpha^{-1}(\rho)\right)=\alpha^{-1}(s(\rho))=\alpha^{-1}\left(r\left(\beta_{2}\right)\right)=s\left(\beta_{2}\right) .
$$

So each $\rho \in \Lambda^{e_{2}}$ determines a commuting square $\rho \beta_{2}=\beta_{1} \alpha^{-1}(\rho)$ and every commuting square $\lambda$ in $\Lambda$ has this form with $\rho:=\left.\lambda\right|_{T\left(e_{2}\right)}$.

Every commuting square in $\left(R \Lambda \times_{\alpha} \mathbb{Z}\right)^{(1,1)}$ has the form $(\rho, 1)$ for some $\rho \in \Lambda^{e_{2}}$ and has factorizations

$$
(\rho, 0)(s(\rho), 1)=(r(\rho), 1)\left(\alpha^{-1}(\rho), 0\right) .
$$

We will show that $\phi$ maps the commuting square $\lambda \in \Lambda^{(1,1)}$ to the commuting square $\left(\left.\lambda\right|_{T\left(e_{2}\right)}, 1\right)$ in $R \Lambda \times_{\alpha} \mathbb{Z}$. Let $\rho:=\left.\lambda\right|_{T\left(e_{2}\right)}$. In pictures,

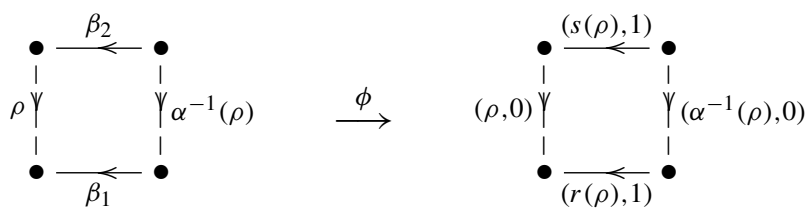

We have $\phi_{2}(\rho)=(\rho, 0)$ and $\phi_{2}\left(\alpha^{-1}(\rho)\right)=\left(\alpha^{-1}(\rho), 0\right)$ by definition, and $\phi_{1}\left(\beta_{2}\right)=\left(r\left(\beta_{2}\right), 1\right)=(s(\rho), 1)$ and $\phi_{1}\left(\beta_{1}\right)=\left(r\left(\beta_{1}\right), 1\right)=(r(\rho), 1)$. So $\phi(\lambda)$ has factorizations $(\rho, 0)(s(\rho), 1)=(r(\rho), 1)\left(\alpha^{-1}(\rho), 0\right)$ which gives $\phi(\lambda)=(\rho, 1)=$ $\left(\left.\lambda\right|_{T\left(e_{2}\right)}, 1\right)$.

\section{The $C^{*}$-algebras of domino graphs}

We now use what we know about the combinatorics of dominoes to describe the $C^{*}$ algebra of a domino graph $\Lambda(n, q, t)$. If $n=1$ or $(n, q, t)=(2,2,0)$, then $\Lambda(n, q, t)$ is a product 2-graph $E \times F$, and $C^{*}(\Lambda(n, q, t))$ is isomorphic to $C^{*}(E) \otimes C^{*}(F)$. For $n=1$, both $E$ and $F$ consist of a single loop, both $C^{*}(E)$ and $C^{*}(F)$ are isomorphic to $C(\mathbb{T})$, and $C^{*}(\Lambda(1, q, t))$ is isomorphic to $C(\mathbb{T}) \otimes C(\mathbb{T})=C\left(\mathbb{T}^{2}\right)$. For $(n, q, t)=(2,2,0), E$ is isomorphic to $K_{1}$ and $F$ to $K_{2}$, and $C^{*}(\Lambda(2,2,0))$ is isomorphic to $C(\mathbb{T}) \otimes C^{*}\left(K_{2}\right)=C(\mathbb{T}) \otimes \mathcal{O}_{2}$.

The remaining cases are handled by the following theorem.

THEOREM 5.1. Suppose that $(n, q, t)$ is a set of basic data with $n \geq 2$ and $(n, q, t) \neq$ $(2,2,0)$, and let $\alpha$ be the action of $\mathbb{Z}$ on $R \Lambda$ described in Proposition 4.6. Let $\mathcal{O}_{\Lambda^{0}}:=C^{*}\left(t_{v}: v \in \Lambda^{0}\right)$ be the Cuntz algebra of the finite set $\Lambda^{0}=\Lambda^{0}(n, q, t)$, and let $\gamma$ be the automorphism of $\mathcal{O}_{\Lambda^{0}}$ such that $\gamma\left(t_{v}\right)=t_{\alpha_{1}(v)}$. Then $\gamma$ has order $n$, 
hence induces an action $\gamma$ of $Z / n \mathbb{Z}$ on $\mathcal{O}_{\Lambda^{0}}$, and there is an isomorphism $\Theta$ of $C^{*}(\Lambda(n, q, t))$ onto the mapping cylinder

$$
M\left(\hat{\gamma}_{e^{2 \pi i / n}}\right)=\left\{f \in C\left([0,1], \mathcal{O}_{\Lambda^{0}} \times_{\gamma}(\mathbb{Z} / n \mathbb{Z})\right): f(1)=\hat{\gamma}_{e^{2 \pi i / n}}(f(0))\right\}
$$

such that

$$
\begin{array}{ll}
\Theta\left(s_{v}\right)(t)=i_{\mathcal{O}_{\Lambda^{0}}}\left(t_{v} t_{v}^{*}\right) & \text { for } v \in \Lambda^{0}, \\
\Theta\left(s_{e}\right)(t)=e^{2 \pi i t / n} i_{\mathcal{O}_{\Lambda^{0}}}\left(t_{r(e)} t_{r(e)}^{*}\right) i_{\mathbb{Z} / n \mathbb{Z}}(1+n \mathbb{Z}) & \text { for } e \in \Lambda^{e_{1},}, \\
\Theta\left(s_{f}\right)(t)=i_{\mathcal{O}_{\Lambda^{0}}}\left(t_{r(f)} t_{S(f)} t_{s(f)}^{*}\right) & \text { for } f \in \Lambda^{e_{2}} .
\end{array}
$$

The crossed product $\mathcal{O}_{\Lambda^{0}} \times_{\gamma}(\mathbb{Z} / n \mathbb{Z})$ is simple and the primitive ideal space of $C^{*}(\Lambda(n, q, t))$ is homeomorphic to $\mathbb{T}$.

PROOF. Lemma 4.4 implies that the permutation $\sigma^{-1}=\left.\alpha_{1}\right|_{\Lambda^{0}}$ has order $n$, and hence so does the induced automorphism $\gamma$ of $\mathcal{O}_{\Lambda^{0}}$. In Proposition 4.6 (and specifically in equation (4.3)) we constructed an isomorphism $\phi$ of $\Lambda(n, q, t)$ onto the crossed product $R \Lambda \times_{\alpha} \mathbb{Z}$, and this induces an isomorphism

$$
\theta_{1}: C^{*}(\Lambda) \rightarrow C^{*}\left(R \Lambda \times_{\alpha} \mathbb{Z}\right)
$$

Proposition 3.1 of [7] implies that the action $\alpha$ of $\mathbb{Z}$ on $R \Lambda$ induces an action $\tilde{\alpha}$ of $\mathbb{Z}$ on the $C^{*}$-algebra $C^{*}(R \Lambda)$ such that $\tilde{\alpha}_{m}\left(s_{\lambda}\right)=s_{\alpha_{m}(\lambda)}$ for $\lambda \in \Lambda$ and $m \in \mathbb{Z}$, and [7, Theorem 3.5] gives us an isomorphism

$$
\theta_{2}: C^{*}\left(R \Lambda \times_{\alpha} \mathbb{Z}\right) \rightarrow C^{*}(R \Lambda) \times_{\tilde{\alpha}} \mathbb{Z},
$$

which is characterized by $\theta_{2}\left(s_{(\mu, m)}\right)=i_{A}\left(s_{\mu}\right) i_{\mathbb{Z}}(m)$. Since the generator is induced by a permutation of order $n, \tilde{\alpha}$ factors through an an action $\beta$ of $\mathbb{Z} / n \mathbb{Z}$ such that $\beta_{m+n \mathbb{Z}}\left(s_{e}\right)=s_{\alpha_{m}(e)}$, and the Olesen-Pedersen theorem in the form of Corollary 3.3 gives us an isomorphism

$$
\theta_{3}: C^{*}(R \Lambda) \times_{\tilde{\alpha}} \mathbb{Z} \rightarrow M\left(\hat{\beta}_{e^{2 \pi i / n}}\right),
$$

where $\hat{\beta}$ is the dual action of $C_{n}$ on the crossed product $C^{*}(R \Lambda) \times \beta(\mathbb{Z} / n \mathbb{Z})$.

Next we recall from Proposition 4.2 that $R \Lambda$ is the complete directed graph $K_{\Lambda^{0}}$ with vertex set $\Lambda^{0}$, which is the dual of the graph $E_{\Lambda^{0}}$ with one vertex and the edges parameterized by $\Lambda^{0}$. Write $\left\{t_{v}: v \in \Lambda^{0}\right\}$ for a universal Cuntz-Krieger family which generates $\mathcal{O}_{\Lambda^{0}}:=C^{*}\left(E_{\Lambda^{0}}\right)$. Then we can deduce from [21, Corollary 2.6], for example, that there is an isomorphism $\psi$ of $C^{*}(R \Lambda)$ onto $\mathcal{O}_{\Lambda^{0}}$ which carries the projections $p_{v}$ into $t_{v} t_{v}^{*}$ and the partial isometries $s_{e}$ into $t_{r(e)} t_{S(e)} t_{s(e)}^{*}$. The inverse $\psi^{-1}$ takes $t_{v}$ to $T_{v}:=\sum_{r(e)=v} s_{e}$, and hence we have

$$
\beta\left(\psi^{-1}\left(t_{v}\right)\right)=\sum_{r(e)=v} \beta\left(s_{e}\right)=\sum_{r(e)=v} s_{\alpha(e)}=\sum_{r(f)=\alpha_{1}(v)} s_{f}=T_{\alpha_{1}(v)}=\psi^{-1}\left(\gamma\left(t_{v}\right)\right) .
$$


Therefore $\psi$ induces an isomorphism $\psi \times$ id of $C^{*}(R \Lambda) \times_{\beta}(\mathbb{Z} / n \mathbb{Z})$ onto $\mathcal{O}_{\Lambda^{0}} \times_{\gamma}(\mathbb{Z} / n \mathbb{Z})$, and composing functions with $\psi \times$ id gives an isomorphism $\theta_{4}$ of $M\left(\hat{\beta}_{e^{2 \pi i / n}}\right)$ onto $M\left(\hat{\gamma}_{e^{2 \pi i / n}}\right)$. Since $\beta$ is induced by a transitive permutation of the edges of $E_{\Lambda^{0}}$, we know from Corollary 3.6 that $\mathcal{O}_{\Lambda^{0}} \times_{\beta}(\mathbb{Z} / n \mathbb{Z})$ is simple and that the primitive ideal space is $\mathbb{T}$.

At this stage, we have an isomorphism $\Theta:=\theta_{4} \circ \theta_{3} \circ \theta_{2} \circ \theta_{1}$ of $C^{*}(\Lambda)$ onto $M\left(\hat{\gamma}_{e^{2 \pi i / n}}\right)$, and we need to check that $\Theta$ does the right thing on generators. For $v \in \Lambda^{0}$,

$$
\Theta\left(s_{v}\right)=\theta_{4} \circ \theta_{3} \circ \theta_{2}\left(s_{(v, 0)}\right)=\theta_{4} \circ \theta_{3}\left(i_{C^{*}(R \Lambda)}\left(p_{v}\right)\right),
$$

and hence for $t \in[0,1]$, we have

$$
\begin{aligned}
\Theta\left(s_{v}\right)(t) & =\psi \times \operatorname{id}\left(\theta_{3}\left(i_{C^{*}(R \Lambda)}\left(p_{v}\right)\right)\right)(t) \\
& =\psi \times \operatorname{id}\left(\Psi\left(i_{C^{*}(R \Lambda)}\left(p_{v}\right)\right)\left(e^{-2 \pi i t / n}\right)\right) \\
& =\psi \times \operatorname{id}\left(\left(i_{C^{*}(R \Lambda)} \times\left(i_{\mathbb{Z}} \circ q\right)\right)\left(\hat{\delta}_{e^{2 \pi i t / n}}\left(i_{C^{*}(R \Lambda)}\left(p_{v}\right)\right)\right),\right.
\end{aligned}
$$

where $\delta:=\beta \circ q$ is the action of $\mathbb{Z}$ inflated from $\beta: \mathbb{Z} / n \mathbb{Z} \rightarrow$ Aut $C^{*}(R \Lambda)$. Since the dual action fixes the range of $i_{C^{*}(R \Lambda)}$, we have

$$
\Theta\left(p_{v}\right)(t)=\psi \times \operatorname{id}\left(\left(i_{C^{*}(R \Lambda)} \times\left(i_{\mathbb{Z}} \circ q\right)\right)\left(i_{C^{*}(R \Lambda)}\left(p_{v}\right)\right)\right)=i_{\mathcal{O}_{\Lambda^{0}}}\left(t_{v} t_{v}^{*}\right),
$$

which is (5.1). For $f \in \Lambda^{e_{2}}$, we have $\Theta\left(s_{f}\right)=\theta_{4} \circ \theta_{3}\left(i_{C^{*}(R \Lambda)}\left(s_{f}\right)\right)$, and a calculation just like (5.7) gives (5.3). Finally, for $e \in \Lambda^{e_{1}}$, we have

$$
\Theta\left(s_{e}\right)=\theta_{4} \circ \theta_{3} \circ \theta_{2}\left(s_{(r(e), 1)}\right)=\theta_{4} \circ \theta_{3}\left(i_{C^{*}(R \Lambda)}\left(p_{r(e)}\right) i_{\mathbb{Z}}(1)\right),
$$

and hence for $t \in[0,1]$, we have

$$
\begin{aligned}
\Theta\left(s_{e}\right) & =\psi \times \operatorname{id}\left(\left(i_{C^{*}(R \Lambda)} \times\left(i_{\mathbb{Z}} \circ q\right)\right)\left(\hat{\delta}_{e^{2 \pi i t / n}}\left(i_{C^{*}(R \Lambda)}\left(p_{r(e)}\right) i_{\mathbb{Z}}(1)\right)\right)\right) \\
& =\psi \times \operatorname{id}\left(\left(i_{C^{*}(R \Lambda)} \times\left(i_{\mathbb{Z}} \circ q\right)\right)\left(i_{C^{*}(R \Lambda)}\left(p_{r(e)}\right) e^{2 \pi i t / n} i_{\mathbb{Z}}(1)\right)\right) \\
& =\psi \times \operatorname{id}\left(i_{C^{*}(R \Lambda)}\left(p_{r(e)}\right) e^{2 \pi i t / n} i_{\mathbb{Z} / n \mathbb{Z}}(1+n \mathbb{Z})\right) \\
& =e^{2 \pi i t / n} i_{\mathcal{O}_{\Lambda^{0}}}\left(t_{r(e)} t_{r(e)}^{*}\right) i_{\mathbb{Z} / n \mathbb{Z}}(1+n \mathbb{Z}) .
\end{aligned}
$$

REMARK 5.2. It is intriguing that the other family of periodic 2-graphs whose algebras have been analysed also have $C^{*}$-algebras with primitive ideal space $\mathbb{T}$ (see [4, Section 5]). The graphs in [4] have just one vertex, and the skeleton admits many possible families $C$ of commuting squares; for domino graphs, the skeleton admits a unique family $C$ of commuting squares.

REMARK 5.3. Since the group $\mathbb{Z} / n \mathbb{Z}$ is finite, Theorem 4.1 of [15] implies that the action $\gamma$ of $\mathbb{Z} / n \mathbb{Z}$ on $\mathcal{O}_{\Lambda^{0}}$ in Theorem 5.1 is proper in the sense of Rieffel [24]; then, since $\mathcal{O}_{\Lambda^{0}} \times_{\gamma}(\mathbb{Z} / n \mathbb{Z})$ is simple, [24, Corollary 1.7] implies that $\mathcal{O}_{\Lambda^{0}} \times_{\gamma}(\mathbb{Z} / n \mathbb{Z})$ is Morita equivalent to the fixed-point algebra $\mathcal{O}_{\Lambda^{0}}^{\gamma}$. However, the underlying action on $R \Lambda$ is not free on any $R \Lambda^{n}$, so the discussion in [15, Section 4] suggests that it may be hard to get useful information about this fixed-point algebra (examples there show that it need not be the $C^{*}$-algebra of the quotient graph, for example). 


\section{6. $K$-theory}

In [18, Section 7] we conjectured, based on the numerical evidence in [18, Table 1], that $K_{0}\left(C^{*}(\Lambda)\right)$ and $K_{1}\left(C^{*}(\Lambda)\right)$ are cyclic groups of the same order. In this section we verify this for domino graphs using the identification of $C^{*}(\Lambda)$ as a crossed product.

Proposition 6.1. If $(n, q, t)$ is basic data, then for $i=0$ and $i=1$,

$$
K_{i}\left(C^{*}(\Lambda(n, q, t))\right)= \begin{cases}\mathbb{Z}^{2} & \text { if } n=1 \\ 0 & \text { if }(n, q, t)=(2,2,0) \\ \mathbb{Z} /\left(q^{n-1}-1\right) \mathbb{Z} & \text { otherwise. }\end{cases}
$$

PROOF. In the first case $C^{*}(\Lambda)=C(\mathbb{T}) \otimes C(\mathbb{T})$ by Theorem 5.1. As $K_{i}(C(\mathbb{T}))=\mathbb{Z}$ [27, p. 234], the $K$-groups of $C(\mathbb{T})$ are torsion-free and the Künneth formula [28] gives

$$
\begin{aligned}
K_{0}(C(\mathbb{T}) \otimes C(\mathbb{T})) & =K_{0}(C(\mathbb{T})) \otimes K_{0}(C(\mathbb{T})) \oplus K_{1}(C(\mathbb{T})) \otimes K_{1}(C(\mathbb{T})) \\
& =\mathbb{Z} \otimes \mathbb{Z} \oplus \mathbb{Z} \otimes \mathbb{Z}=\mathbb{Z}^{2} \\
K_{1}(C(\mathbb{T}) \otimes C(\mathbb{T})) & =K_{0}(C(\mathbb{T})) \otimes K_{1}(C(\mathbb{T})) \oplus K_{1}(C(\mathbb{T})) \otimes K_{0}(C(\mathbb{T})) \\
& =\mathbb{Z} \otimes \mathbb{Z} \oplus \mathbb{Z} \otimes \mathbb{Z}=\mathbb{Z}^{2}
\end{aligned}
$$

In the second case $C^{*}(\Lambda)=C(\mathbb{T}) \otimes \mathcal{O}_{2}$ by Theorem 5.1, and since $K_{i}\left(\mathcal{O}_{2}\right)=0$ [27, p. 234] the Künneth formula gives $K_{i}\left(C(\mathbb{T}) \otimes \mathcal{O}_{2}\right)=0$. Otherwise, we have $C^{*}(\Lambda) \cong \mathcal{O}_{\Lambda^{0}} \times_{\tilde{\alpha}} \mathbb{Z}$. Since $\mathcal{O}_{\Lambda^{0}}$ is a Cuntz algebra with $q^{n-1}$ generators, we deduce from $[27$, p. 234], for example, that

$$
K_{1}\left(\mathcal{O}_{\Lambda^{0}}\right)=0 \quad \text { and } \quad K_{0}\left(\mathcal{O}_{\Lambda^{0}}\right)=\mathbb{Z} /\left(q^{n-1}-1\right) \mathbb{Z},
$$

where $K_{0}\left(\mathcal{O}_{\Lambda^{0}}\right)$ is generated by the class [1] of the identity. Thus the PimsnerVoiculescu sequence [20] for this crossed product reduces to

$$
0 \longrightarrow K_{1}\left(\mathcal{O}_{\Lambda^{0}} \times_{\tilde{\alpha}} \mathbb{Z}\right) \longrightarrow K_{0}\left(\mathcal{O}_{\Lambda^{0}}\right) \stackrel{\mathrm{id}-\tilde{\alpha}_{*}}{\longrightarrow} K_{0}\left(\mathcal{O}_{\Lambda^{0}}\right) \longrightarrow K_{0}\left(\mathcal{O}_{\Lambda^{0}} \times_{\tilde{\alpha}} \mathbb{Z}\right) \longrightarrow 0
$$

and we have id $-\tilde{\alpha}_{*}=0$ because $\tilde{\alpha}_{*}(1)=1$. So both $K_{0}\left(C^{*}(\Lambda)\right)=K_{0}\left(\mathcal{O}_{\Lambda^{0}} \times_{\tilde{\alpha}} \mathbb{Z}\right)$ and $K_{1}\left(C^{*}(\Lambda)\right)=K_{1}\left(\mathcal{O}_{\Lambda^{0}} \times_{\tilde{\alpha}} \mathbb{Z}\right)$ are isomorphic to $\mathbb{Z} /\left(q^{n-1}-1\right) \mathbb{Z}$.

\section{Appendix A. Necklaces and Lyndon words}

A word is a finite or infinite sequence of symbols from a finite set $A$ called the alphabet. Subsets of the set $A^{*}$ of all finite words are called languages and we write $A^{n}$ for the words of length $n$. The product of two words $u$ and $v$ is their concatenation $u v$, and for $k \in \mathbb{N}$ the $k$ th power of $u$ is $u^{k}$.

Consider the alphabet $A=\{0,1, \ldots, q-1\}$, and define $\rho: A^{n} \rightarrow A^{n}$ by $\rho\left(a_{1} \cdots a_{n}\right)=a_{2} \cdots a_{n} a_{1}$. Then the permutation group $\langle\rho\rangle$ acts on $A^{n}$, and the equivalence classes under rotation are known as necklaces of length $n$, so called 
because a necklace of length $n$ can be visualized as a regular $n$-gon where the corners represent the 'beads', each designated one of $q$ colours. For example, if $q=2$ and $n=6$, the class of 011011 is the necklace $[011011]=\{011011,101101,110110\}$ and is drawn as

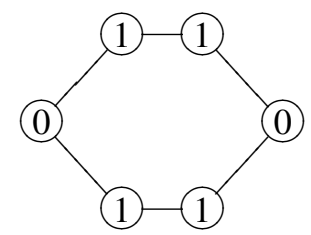

The period of a necklace $[a]$ is the smallest $d \in \mathbb{N}$ such that $\rho^{d}(a)=a$, and it must divide the length of the necklace. A necklace with period equal to its length is called aperiodic and its lexicographic least representative is known as a Lyndon word (originally called standard lexicographic sequences in [14]). For example, 000011 and 000001 are Lyndon words of length 6. For every necklace $[a]$ of length $n$ and period $d$ there is a unique subword $b$ of length $d$ such that $\left[b^{n / d}\right]=[a]$; we call $b$ the Lyndon subword of [a]. For example, the necklace [011011] in (A.1) has period 3 and Lyndon subword 011.

We identify the alphabet $A=\{0,1, \ldots, q-1\}$ with the commutative $\operatorname{ring} \mathbb{Z} / q \mathbb{Z}$, and let the trace of a word be the sum of its symbols $\bmod q$. We will often consider the collection $A_{t}^{n}$ of words with length $n$ and trace $t \bmod q$. See Table A1 for the period, trace and Lyndon subword of each binary necklace of length 6 .

By [29, Theorem 1.2] the number of Lyndon words of length $n$ with trace $t$ mod $q$ over the alphabet $\{0,1, \ldots, q-1\}$ is given by

$$
L_{q}(n, t)=\frac{1}{q n} \sum_{\substack{d|n \\ \operatorname{gcd}(d, q)| t}} \operatorname{gcd}(d, q) \mu(d) q^{n / d},
$$

where $\mu$ is the Möbius function.

TABLE A1. The binary necklaces of length 6 .

\begin{tabular}{cccccc}
\hline Necklaces of $A_{0}^{6}$ & Period & Lyndon subword & Necklaces of $A_{1}^{6}$ & Period & Lyndon subword \\
\hline$[000000]$ & 1 & 0 & {$[000001]$} & 6 & 000001 \\
{$[000011]$} & 6 & 000011 & {$[000111]$} & 6 & 000111 \\
{$[000101]$} & 6 & 000101 & {$[001011]$} & 6 & 001011 \\
{$[001001]$} & 3 & 001 & {$[001101]$} & 6 & 001101 \\
{$[001111]$} & 6 & 001111 & {$[010101]$} & 2 & 01 \\
{$[010111]$} & 6 & 010111 & {$[011111]$} & 6 & 011111 \\
{$[011011]$} & 3 & 011 & & & \\
{$[111111]$} & 1 & 1 & & & \\
\hline
\end{tabular}




\section{References}

[1] S. Adji, I. Raeburn and R. Rosjanuardi, 'Group extensions and the primitive ideal spaces of group algebras', Glasg. Math. J. 49 (2007), 81-92.

[2] N. Dang Ngoc, 'Produits croisés restreintes et extensions de groupes', mimeographed notes, Paris 1977.

[3] K. R. Davidson, S. C. Power and D. Yang, 'Atomic representations of rank 2 graph algebras', J. Funct. Anal. 255 (2008), 819-853.

[4] K. R. Davidson and D. Yang, 'Periodicity in rank 2 graph algebras', Canad. J. Math. 61 (2009), 1239-1261.

[5] S. Echterhoff, 'On induced covariant systems', Proc. Amer. Math. Soc. 108 (1990), 703-706.

[6] D. G. Evans, 'On the $K$-theory of higher rank graph $C^{*}$-algebras', New York J. Math. 14 (2008), $1-31$.

[7] C. Farthing, D. Pask and A. Sims, 'Crossed products of $k$-graph algebras by $\mathbb{Z}^{l}$ ', Houston J. Math. 35 (2009), 903-933.

[8] N. J. Fowler and I. Raeburn, 'The Toeplitz algebra of a Hilbert bimodule', Indiana Univ. Math. J. 48 (1999), 155-181.

[9] P. Green, 'The local structure of twisted covariance algebras', Acta Math. 140 (1978), 191-250.

[10] Y. Katayama and H. Takehana, 'On automorphisms of generalized Cuntz algebras', Int. J. Math. 9 (1998), 493-512.

[11] A. Kishimoto, 'Outer automorphisms and reduced crossed products of simple $C^{*}$-algebras', Commun. Math. Phys. 81 (1981), 429-435.

[12] B. Kitchens and K. Schmidt, 'Mixing sets and relative entropies for higher dimensional Markov shifts', Ergod. Th. \& Dynam. Sys. 13 (1993), 705-735.

[13] A. Kumjian and D. Pask, 'Higher rank graph $C^{*}$-algebras', New York J. Math. 6 (2000), 1-20.

[14] R. C. Lyndon, 'On Burnside problem I', Trans. Amer. Math. Soc. 77 (1954), 202-215.

[15] D. Marelli and I. Raeburn, 'Proper actions which are not saturated', Proc. Amer. Math. Soc. 137 (2009), 2273-2283.

[16] D. Olesen and G. K. Pedersen, 'Partially inner $C^{*}$-dynamical systems', J. Funct. Anal. 66 (1986), 263-281.

[17] D. Pask, I. Raeburn, M. Rørdam and A. Sims, 'Rank-two graphs whose $C^{*}$-algebras are direct limits of circle algebras', J. Funct. Anal. 239 (2006), 137-178.

[18] D. Pask, I. Raeburn and N. A. Weaver, 'A family of 2-graphs arising from two-dimensional subshifts', Ergod. Th. \& Dynam. Sys. 29 (2009), 1613-1639.

[19] D. Pask and S.-J. Rho, 'Some intrinsic properties of simple graph $C^{*}$-algebras', in: Proceedings of the Conference on Operator Algebras and Mathematical Physics, Constanţa, 2001 (Theta Foundation, Bucharest, 2003), pp. 325-340.

[20] M. V. Pimsner and D. Voiculescu, 'Exact sequences for $K$-groups and Ext-groups of certain crossproducts of $C^{*}$-algebras', J. Operator Theory 4 (1980), 93-118.

[21] I. Raeburn, Graph Algebras, CBMS Regional Conference Series in Mathematics, 103 (American Mathematical Society, Providence, RI, 2005).

[22] I. Raeburn, A. Sims and T. Yeend, 'Higher-rank graphs and their $C^{*}$-algebras', Proc. Edinb. Math. Soc. 46 (2003), 99-115.

[23] I. Raeburn and D. P. Williams, Morita Equivalence and Continuous-Trace C*-Algebras, Mathematical Surveys and Monographs, 60 (American Mathematical Society, Providence, RI, 1998).

[24] M. A. Rieffel, 'Proper actions of groups on $C^{*}$-algebras', in: Mappings of Operator Algebras, Progress in Mathematics, 84 (Birkhäuser, Boston, MA, 1990), pp. 141-182.

[25] D. I. Robertson and A. Sims, 'Simplicity of $C^{*}$-algebras associated to higher rank graphs', Bull. London Math. Soc. 39 (2007), 337-344.

[26] G. Robertson and T. Steger, 'Affine buildings, tiling systems and higher rank Cuntz-Krieger algebras', J. reine angew. Math. 513 (1999), 115-144.

[27] M. Rørdam, F. Larsen and N. J. Lausten, An Introduction to $K$-Theory for $C^{*}$-Algebras, London Mathematical Society Student Texts, 49 (Cambridge University Press, Cambridge, 2000). 
[28] J. Rosenberg and C. Schochet, 'The Künneth theorem and the universal coefficent theorem for Kasparov's generalised K-functor', Duke Math. J. 55 (1987), 431-474.

[29] F. Ruskey, C. R. Miers and J. Sawada, 'The number of irreducible polynomials and Lyndon words with given trace', SIAM J. Discrete Math. 41 (2001), 240-245.

[30] K. Schmidt, Dynamical Systems of Algebraic Origin, Progress in Mathematics, 128 (Birkhäuser, Basel, 1995).

[31] A. Skalski and J. Zacharias, 'Entropy of shifts on higher-rank graph $C^{*}$-algebras', Houston J. Math. 34 (2008), 269-282.

[32] D. P. Williams, Crossed Products of $C^{*}$-Algebras, Mathematical Surveys and Monographs, 134 (American Mathematical Society, Providence, RI, 2007).

DAVID PASK, School of Mathematics and Applied Statistics, University of Wollongong, NSW 2522, Australia

e-mail: dpask@uow.edu.au

IAIN RAEBURN, School of Mathematics and Applied Statistics, University of Wollongong, NSW 2522, Australia

e-mail: raeburn@uow.edu.au

NATASHA A. WEAVER, School of Mathematical and Physical Sciences, University of Newcastle, NSW 2308, Australia

e-mail: Tash.Weaver@gmail.com 